\title{
Low radon exposures \& lung cancer risk: joint analysis of Czech, French \& Beaverlodge uranium miners
}

\author{
Rachel Lane ${ }^{1,2,{ }^{*}}$, Ladislav Tomášek ${ }^{3}$, Lydia Zablotska ${ }^{4}$, Estelle Rage ${ }^{5}$, Franco Momoli ${ }^{1,6}$, \\ and Julian Little ${ }^{1}$ \\ ${ }^{1}$ School of Epidemiology and Public Health, Faculty of Medicine, University of Ottawa; Room 101, \\ 600 Peter Morand Crescent, Ottawa, ON, Canada, K1G $5 Z 3$ \\ ${ }^{2}$ Canadian Nuclear Safety Commission (CNSC), 280 Slater Street, P.O. Box 1046, Station B. Ottawa, \\ ON, Canada, K1P 5S9 \\ ${ }^{3}$ National Radiation Protection Institute (SURO), Bartoskova 28, CZ-140 00 Prague, Czech Republic \\ ${ }^{4}$ Department of Epidemiology and Biostatistics, School of Medicine, University of California, San \\ Francisco, 550 16th St, San Francisco, CA 94158, USA \\ ${ }^{5}$ Institute for Radiological Protection and Nuclear Safety, B.P. 17, F-92262 Fontenay-aux-Roses \\ Cedex, France \\ ${ }^{6}$ Centre for Practice-Changing Research, Ottawa Hospital Research Institute, Children's Hospital of \\ Eastern Ontario Research Institute; 401 Smyth Rd, Ottawa, ON, Canada, K1H 8L1
}

It is well established that high radon exposures increase the risk of lung cancer mortality. The effects of low occupational exposures and the factors that confound and modify this risk are not clear and are needed to inform current radiation protection of miners. The risk of lung cancer mortality at low radon exposures ( $<100$ working level months) was assessed in the joint cohort analysis of Czech, French and Canadian uranium miners, employed in 1953 or later.

Statistical analysis was based on linear Poisson regression modeling with grouped cohort survival data. Two sensitivity analyses were used to assess potential confounding from tobacco smoking.

A statistically significant linear relationship between radon exposures and lung cancer mortality was found. The excess relative risk per working level month was $0.022(95 \%$ confidence interval: $0.013-0.034$ ), based on 408 lung cancer deaths and 394,236 personyears. Miners who received radon exposures 5-14 years previously and miners aged $<55$ years had the greatest risk of lung cancer mortality. These risks decreased with increasing time since exposure and attained age. No effect of exposure rate, separate from measures of cumulative exposure, was observed at low radon exposure levels. The potential confounding effect of tobacco smoking was estimated to be small and did not substantially change the radon-lung cancer mortality risk estimates.

This joint cohort analysis provides strong evidence for an increased risk of lung cancer mortality from low occupational radon exposures. The results suggest radiation protection measures continue to be important among current uranium miners.

* Corresponding author: rachel.lane@canada.ca 